\title{
LA-UR-93- $93-3101$
}

Title:

PERFORMANCE OF THE HIGH BRIGHTNESS LINAC

FOR THE ADVANCED FREE ELECTRON LASER

INITIATIVE AT LOS ALAMOS

Author(s):

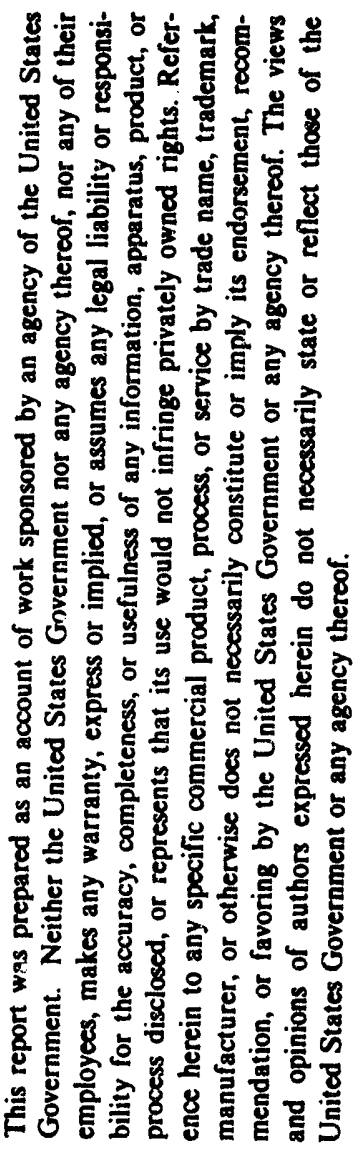

Submitted to:

R. L. Sheffield, R. H. Austin, K. D. C. Chan, S. M. Gierman, J. M. Kinross-Wright, S. H. Kong, D. C. Nguyen, S. J. Russell, and C. A. Timmer
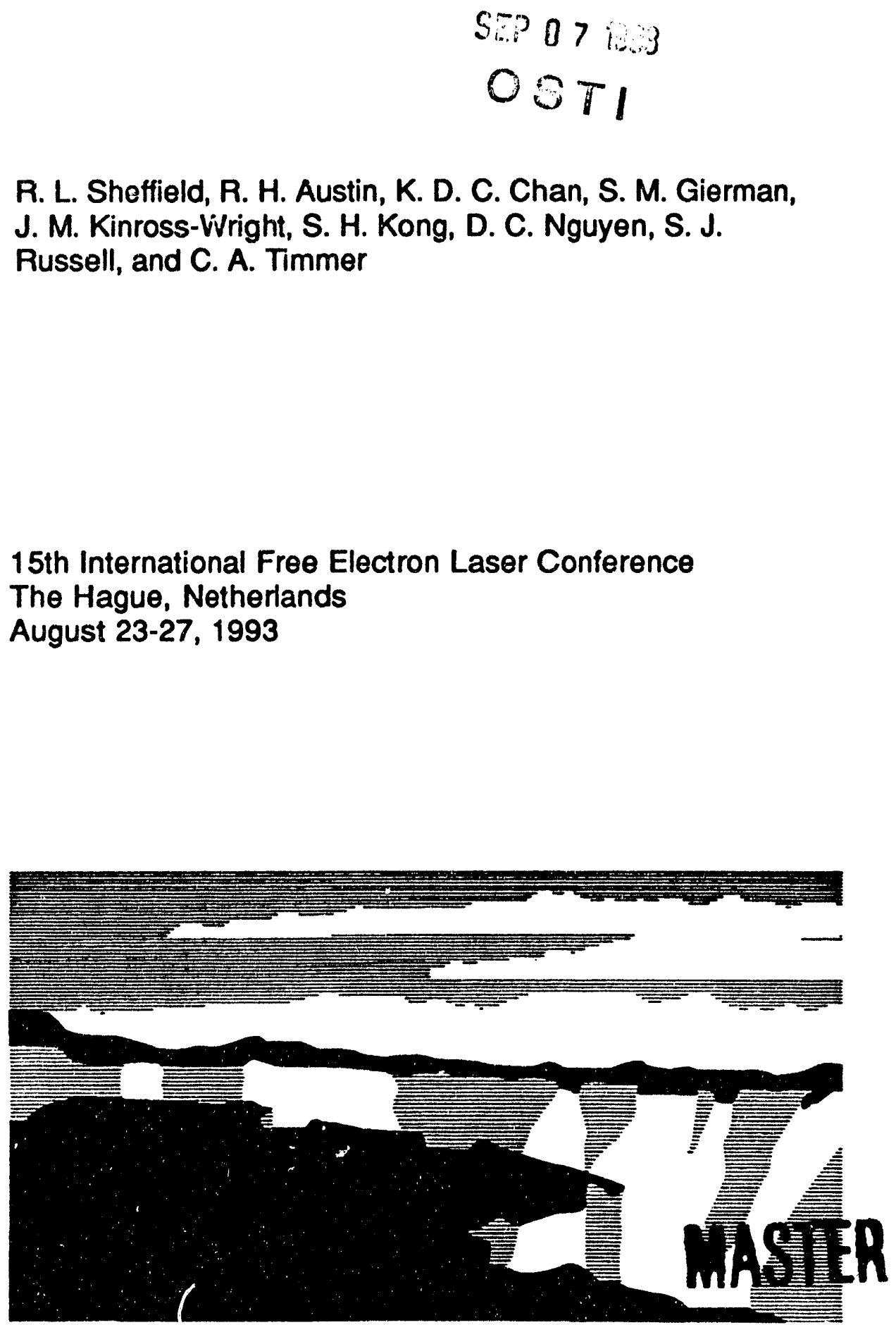

Los Alamos National Laboratory, an aftirmative actionequal opportunity employer, is operated by the University of California lor the U.S. Department of Energy under contract W-7405-ENG.36. By acceptance of this artide, the publisher recognizes that the U.S. Government retains a nonexclusive, royalty-free license is publish or reproduce the published form of this contribution, or to allow others to do so, for U.S. Goinrnment purposes. The Loe Alamos National Laboratory requests that the publisher identify this artide as work pertormed under the auspices of the U.S. Drzartment of Energy. 
Performance of the High Brightness LINAC for the Advanced Free-Electron $I$ aser Initiative at Los Alamos*, R. L. Sheffield, R. H. Austin, K. D. C. Chan, S. M. Gierman, J. M. Kinross-Wright, S. H. Kong, D. C. Nguyen, S. J. Russell, and C. A. Timmer, Los Alamos National Laboratory, Los Alamos, NM 87545

\begin{abstract}
The AFEL accelerator has produced beams of greater than $2 \times 10^{12} \mathrm{~A} / \mathrm{m}^{2}$ at $1 \mathrm{nC}$ (brightness $=2 * \mathrm{I}$ $\varepsilon^{2}$, with I greater than $100 \mathrm{~A}$ and $\varepsilon$ of than $2 \pi \mathrm{mm}$-mrad normalized ms emittance). The $1300 \mathrm{MHz}$ standing-wave accelerator uses on-axis coupling cells. The electron source is a photoinjector with a $\mathrm{CsK}_{2} \mathrm{Sb}$ photocathode. The photoinjector is an integral part of a single 11-cell accelerator structure. The accelerator operates between 12 and $18 \mathrm{MeV}$. The beam emittance growth in the accelerator is minimized by using a photoinjector, a focusing solenoid to correct the emittance growth due to space charge, and a special design of the coupling slots between accelerator cavities to minimize quadrupole effects. This paper describes the experimental results and compares those results with PARMELA simulation. The simulation code PARMELA was modified for this effort. This modified version uses SUPERFISH files for the accelerator cavity fields, MAFIA files for the fields due to the coupling slots in the accelerator cells, and POISSON files for the solenoid field in the gun region.
\end{abstract}

\title{
1. INTRODUCTION
}

An accelerator design that produces a very bright electron beam has ieen constructed through the Advanced Free-Electron Laser Initiative (AFELI) ${ }^{1}$ at Los Alamos National Laboratory. State-of-theart components were incorporated so that the FEL system will be compact, robust, and user friendly.

The design goals for the accelerator were: greater $2 \mathrm{nC}$ charge per micropulse and an effective emittance of less than $5 \pi \mathrm{mm}$-mrad. Simple design is accomplished by using a single radio-frequency feed to drive the entire accelerator structure. The accelerator (Fig. 1) design has the following features: maximum $20-\mathrm{MeV}$ output energy, maximum average cell gradients of $22 \mathrm{MeV} / \mathrm{m}$, up to $100-\mathrm{Hz}$ repetition rate, up to $30-\mu$ s long macropulses, 8- to 20-ps long micropulses, and liquid-nitrogen

\footnotetext{
*Work performed under the auspices of the US D.O.E. and funded by LANL Laboratory Directed Research and Development.
} 
operation capability. The accelerator operates with a $1300-\mathrm{MHz}, 17-\mathrm{MW}$-peak-power klystron. ${ }^{1}$

\section{SIMULATIONS}

The following definition is used for the normalized ms emittance in the simulations,

$$
\varepsilon_{n}=\beta \gamma \varepsilon_{x}=\pi \beta \gamma\left[<x^{2}><x^{\prime 2}>-<x \cdot x^{\prime}>^{2}\right]^{1 / 2} \text {, }
$$

where $\gamma$ is the relativistic factor, $\beta$ is the particle velocity divided by the speed of light, $x$ is the transverse beam size, $x^{\prime}$ is the transverse beam divergence, and $\varepsilon_{x}$ is the unnormalized emittance. The emittance is calculated in two ways. The "full" emittance is calculated in the conventional manner by integrating over the entire electron distribution of the micropulse in time and space.

The "slice" emittance is calculated by dividing a micropulse into slices in time equal to a slippage length (the slippage length is the wavelength of the light times the number of wiggler periods). To ensure enough particles are in a slice to give reasonable statistics, the smallest time slice is limited to a minimum $1 \%$ of the total pulse length (4000 particles were used in the simulations). We calculate the slice emittance because the electrons are not matched to the wiggler over the entire pulse, but only for the middle portion (in time) of the pulse. Temporal mixing can occur downstream of the accelerator and before the wiggler, degrading the slice emittance. Therefore, the use of slice emittance is only valid at the location of the wiggler.

\subsection{Emittance Compensation}

Surrounding the first few cells is a large focusing solenoid. The use of a solenoid to correct emittance growth caused by space charge has been discussed in detail in several papers. ${ }^{2} \mathrm{~A}$ brief explanation of emittance compensation follows. A representative configuration is shown in Fig. 2. At the cathode, the electron bunch emittance is determined by the cathode's intrinsic thermal emittance (position 1 in Fig. 2). As an electron bunch leaves the cathode, the bunch expands radially because of radial space charge forces. Since the space charge force acts continuously on the bunch, no single discrete lens can compensate for the distortion of the distribution in phase space (position 2 in Fig. 2) as the beam propagates. However, a simple le is can be used to focus the bunch (position 3 in Fig. 
2). Then, to the first order, the same forces that acted on the bunch during expansion are present while the bunch is focused (position 4 in Fig. 2). Thus, the emittance growth that has occurred can be significantly reduced by proper lens placement. A unique solenoid design follows from the requirements of minimum emittance growth and simultaneously having the beam focused at a particular axial location. The solenoid design depends on the accelerator gradient, current density, and location of the peak magnetic field with respect to the cathode. The emittance numbers in Fig. 2 are from a typical PARMELA run for a $20 \mathrm{MeV}, 2.3 \mathrm{nC}$ electron pulse. To accurately render the solenoid field profiles, we incorporated the POISSON field maps of the solenoid directly into a modified version of PARMELA.

Using similations, we also computed the effect on the final emittance caused from the cathode thermal effects. As expected, the final emittance is the sum of squares of the final emittance calculated with zero cathode temperature and the cathode's initial intrinsic emittance. An example is shown in Fig. 2.

\subsection{Coupling-slot arrangement}

The standing-wave, $1300-\mathrm{MHz}, \pi / 2$-mode accelerator is designed with on-axis coupling slots. ${ }^{1}$ By incorporating MAFIA field maps of the coupling slots into PARMELA, we found that coupling cells with only two coupling-slots produces a significant quadrupole lens in every accelerator cell. The effect of this lens is very prominent in the first and second accelerator cells. Therefore, it was necessary to take into account the coupling slot geometries to minimize asymmetric focusing in the accelerator.

The coupling-slot design for the AFEL accelerator uses a four-coupling-slot arrangement for the first two cells and a two-coupling-slot configuration for the remaining accelerator cells. Because the four-slot arrangement has no quadrupole component, then the first two cells produce no beam asymmetry. After the beam exits the first two cells, the beam is highly relativistic and the two-slot coupling gives a very small net quadrupole focusing. The coupling-slots arrangement is explained in more detail in Ref. 1.

\subsection{Other design features}

The first cell, a half-cell, is $9 \mathrm{~mm}$ longer than one-half of a standard 1300-Mhz cell. A longer 
injection cell has two advantages. First, the exit phase of the electron bunch depends on the cell length. Since the AFEL linac has a single rf feed, the proper operating phase to minimize energy spread was met by adjusting the first cell length. Second, a longer first cell increases the electron-beam energy at the exit of the first cell. This reduces the space-charge effects and helps improve the final emittance. The exit energy from the first cell is $1.5 \mathrm{MeV}$ instead of $1.0 \mathrm{MeV}$ for a regular half-cell.

\subsection{Beam dependencies}

Thistype of accelerator is unique in that the electron-beam distribution does not mix lcngitudinally. With no mixing, the rms emittance calculation for the full pulse overestimates the effect of beam emittance on the FEL performance. Except for statistical noise caused by the limited number of particles in the simulation, the slice emittance is time independent during the micropulse. However, the emittance of the full pulse is significantly larger. The larger full-pulse emittance is caused from the variation in beam divergence throughout the micropulse. In particular, the front and back of the pulse, which account for a small fraction of the total charge $(<10 \%)$, have ten times the divergence of the middle of the pulse.

The AFEL is designed to minimize components and distances and to increase reliability and ease of use. However, the stability of accelerator operation does depend strongly on a few parameters. The parameters that must be tightly controlled are the cathode radius; the magnitude of the solenoid field in the cathode region; the accelerator phase; and the magnitude of the accelerator fields.

\section{OPERATION OF THE ACCELERATOR}

\subsection{Photocathode}

The eleciron source is a $\mathrm{CsK}_{2} \mathrm{Sb}$ photocathode. The accelerator pressure is maintained at $8 \times 10^{-}$

10 Torr. The beamline directly downstream of the accelerator is at $2 \times 10^{-8}$ Torr. Because of contaminates produced during operation of the rf, the useful operational lifetime of a single cathode, for a minimum $1 \mathrm{nC}$, is 2 days. The accelerator has six cathodes available in a cartridge type system. Thus, the cathode cartridge system was refreshed every two weeks. Typical time to replace a cartridge pack was 15 minutes. After replacement, a 4 to 5 hour bake degassed the components exposed to air (typically done during the night). The time required to exchange one of the cathodes for another 
cathode was less than 5 minutes.

\subsection{Drive Laser}

The drive laser was Nd:YLF. The oscillator produces 50 ps micropulses at $108 \mathrm{MHz}$. The pulses are then compressed with a grating pair and a fiber-optic line to $8 \mathrm{ps.} \mathrm{After} \mathrm{compression,} \mathrm{two} \mathrm{double-}$ pass YLF amplifiers are used to increase the micropulse energy to 25 microjoules. A Pockels Cell is used to obtain macropulses which ranged from a few micropulses to 10 microseconds. A KTP crystal is then used to double the wavelength to $526 \mathrm{~nm}$ with a micropulse energy of 8 microjoules. The gaussian spatial profile of the laser beam is sent through an iris that just lets the middle $40 \%$ of the light pass. This approximately-uniform spatial profile is then imaged onto photocathode.

\subsection{Multipactoring}

The accelerator was initially conditioned for operation from 9 to $16 \mathrm{MeV}$. Since this initial conditioning, the maximum energy has been extended to $18 \mathrm{MeV}$ through the course of normal operation. In March of 1993, after a year of operation, the accelerator began to multipactor. (The emittance measurements discussed in the next section were taken in January). The multipactoring causes a complete field collapse in the accelerator cells. The onset of multipactoring is dependent on the current in the solenoids around the injector end of the accelerator (Fig. 1). The operation of the accelerator was affected in two significant ways. First, the minimum beam energy was limited to 14 $\mathrm{MeV}$ for any useful solenoid field strength (>10 A, typical current for correct matching were $320 \mathrm{~A}$ ). More importantly, we could not adjust the bucking coil (Fig. 1) to zero the field on surface of the cathode, thus leaving a net magnetic field. A non-zero cathode magnetic field gives an increased beam emittance at a rate of $1 \pi \mathrm{mm}$-rad per 6 gauss of residual cathode field. The cause of the multipactoring was reduced to two possibilities. The accelerator was opened and inspected with a bore-scope. We found a $1 \mathrm{~mm}$ sliver of what appeared to be stainless steel firmly attached to the copper wall of the first accelerator cell. The sliver was approximately $2 \mathrm{~cm}$ off-axis and directly below the photocathode. The second possibility was that cesium may have been sputtered onto the cavity surfaces or into the coupling cell. Cesium oxide has a very high secondary emission coefficient and could enable multipactoring. Both possibilities were addressed by the following procedures. First, the sliver was 
removedby wiping with a stiff swap. Second, since cesium and cesium oxide are very soluble, the accelerator was washed with de-ionized water to remove any cesium compounds. These cleaning procedures eliminated the multipactoring.

\section{EXPERIMENTAL RESULTS}

The emittance measurements were taken at an electron beam energy of $13 \mathrm{MeV}$ for $3 \mathrm{nC}$ and 13.5 $\mathrm{MeV}$ for 1 and $2 \mathrm{nC}$. A quadrupole is positioned $27 \mathrm{~cm}$ upstream from an OTR screen. The FWHM of the electron beam was measured as a function of the quadrupole field strength. The $1 \mathrm{nC}$ data set is shown in Fig. 3. Similar sets were obtained for 2 and $3 \mathrm{nC}$ micropulse charges.

The electron beam produced by this accelerator is characterized by a bright temporal core with high-divergence wings at the front and back of the pulse. Very little charge is present in these wings, but, due to their large divergence, the wings significantly increase the rms emittance of the beam as calculated by PARMELA. However, the wings are too tenuous to be observed in the experimental data. Thus the comparison of the measured emittance to the simulation results has to be done on a more detailed level.

The only means to characterize the performance of the accelerator is to also simulate the quadrupole scan with PARMELA and then compare the experimental observable - the beam'sFWHM size. Figure 4 compares the full rms emittance with the slice emittance and the emittance as calculated by using the FWHM measured on spot profiles produced by PARMELA. The emittance as calculated from the FWHM is very close to the slice emittance (the emittance used by the FEL).

The FWHM simulation results for the $1 \mathrm{nC}$ case is given in Fig. 3. The emittance is calculated from the experimental data by first numerically matching the beam spot with a gaussian and then using a non-linear least-square fit on the spot size vs quadrupole field strength. The fit also gives the Twiss parameters of the beam. A difficulty with this technique is that for very low emittance beams the calculated emittance is very sensitive to the screen resolution. The minimum spatial resolution of our screen was $100 \pm 20$ microns. This error leads to a variation of greater than $50 \%$ in the emittance at $1 \mathrm{nC}$. At higher charges the emittance is larger and thus the minimum spot size is larger, decreasing the error in the measured emittance to less than $10 \%$ at $3 \mathrm{nC}$. The emittances for 1,2 , and $3 \mathrm{nC}$ 
calculated from PARMELA are 2, 3, and $4.5 \pi \mathrm{mm}$-mrad and calculated from the experimental data are 1.3 $\pm 0.7,3.0 \pm 0.7$, and $6.5 \pm 0.6 \pi \mathrm{mm}$-mrad for 1,2 , and $3 \mathrm{nC}$, respectively (Fig. 5). The measured emittance is within experimental error of the simulations for 1 and $2 \mathrm{nC}$.

\section{FEL OPERATION}

This April, after beam characterization, the AFEL lased at 5 microns. The AFEL laser performance is discussed in a paper by Dinh Nguyen et al presented at this conference.

\section{REFERENCES}

1. K. C. D. Chan,, R. H. Kraus, J. Ledford, K.L. Meier, R. E. Meyer, D. Nguyen, R. L. Sheffield, F. E. Sigler, L. M. Young, T. S. Wang, W. L. Wilson, and R. L. Wood, Nucl. Inst. Meth. in Phys., A318, 148 (1992). R. L. Sheffield, B. E. Carlsten, and L. M. Young, Proc. Linear Accel. Conf., AECL10728, 1,46 (1992).

2. B. E. Carlsten, Proc. 10th Int. FEL Conf., Jerusalem, Israel, 1988, Nucl. Instr. and Meth., A285 (1989) 313; B. E. Carlsten and R. L. Sheffield, Poc. 1988 Linac Conf., Williamsburg, Va, 1988, CEBAF Report 89-001 (1989) 365; B. E. Carlsten, Poc. 1989 IEEE Part. Accel. Conf., Chicago, IL, IEEE Catalog no. 89CH2669-0 (1989) 313. 


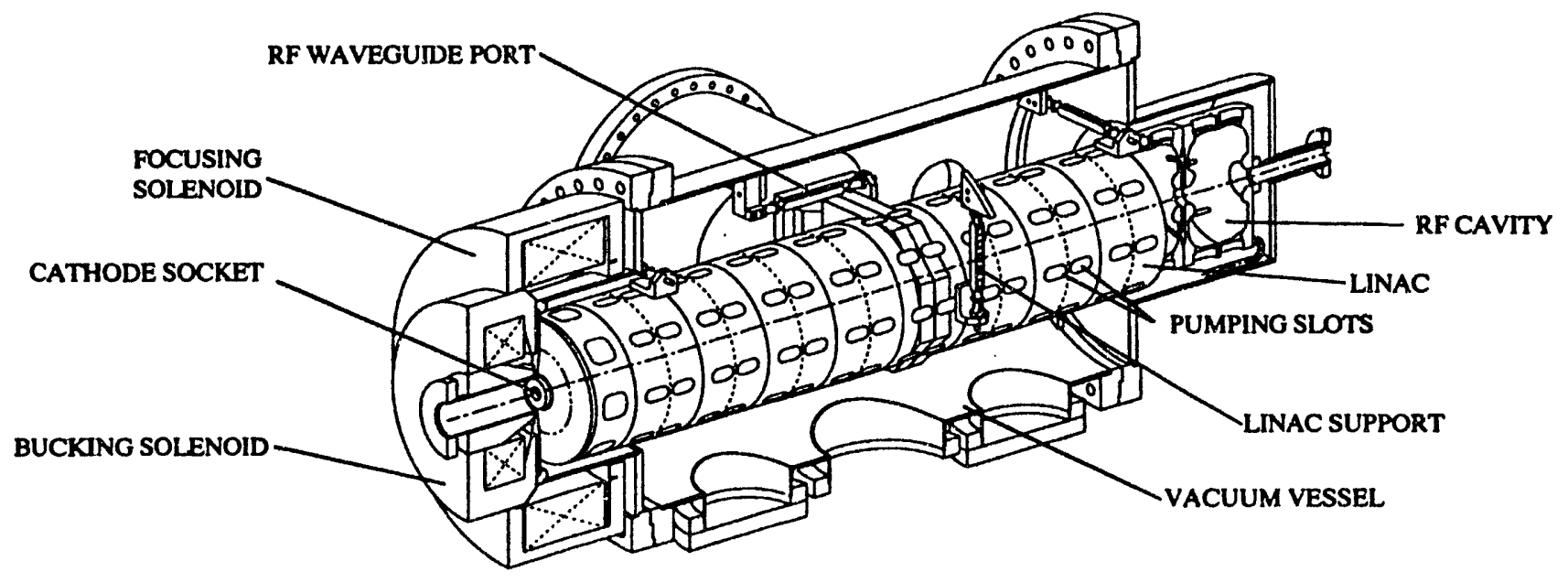

Figure 1 

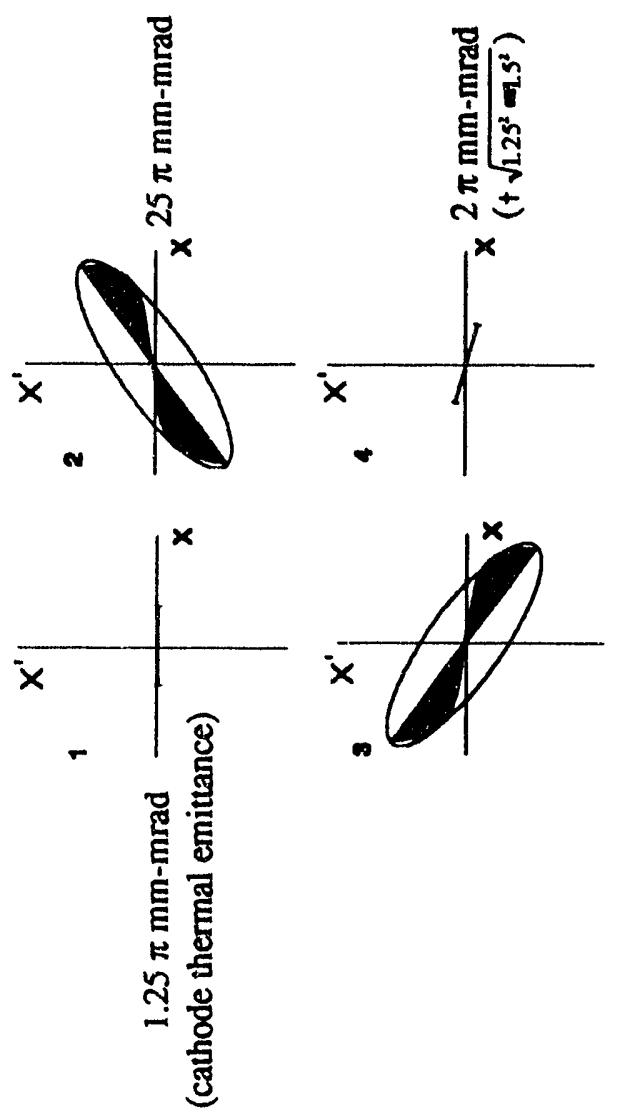

瓷

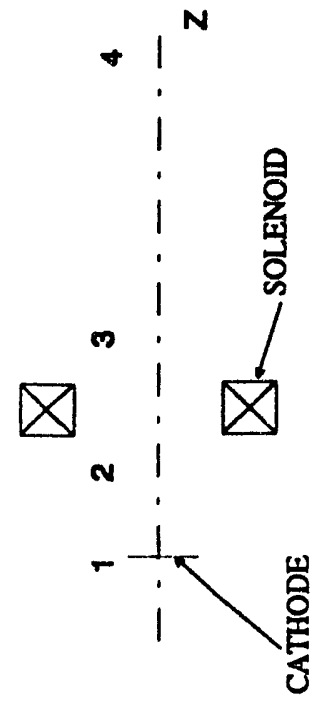




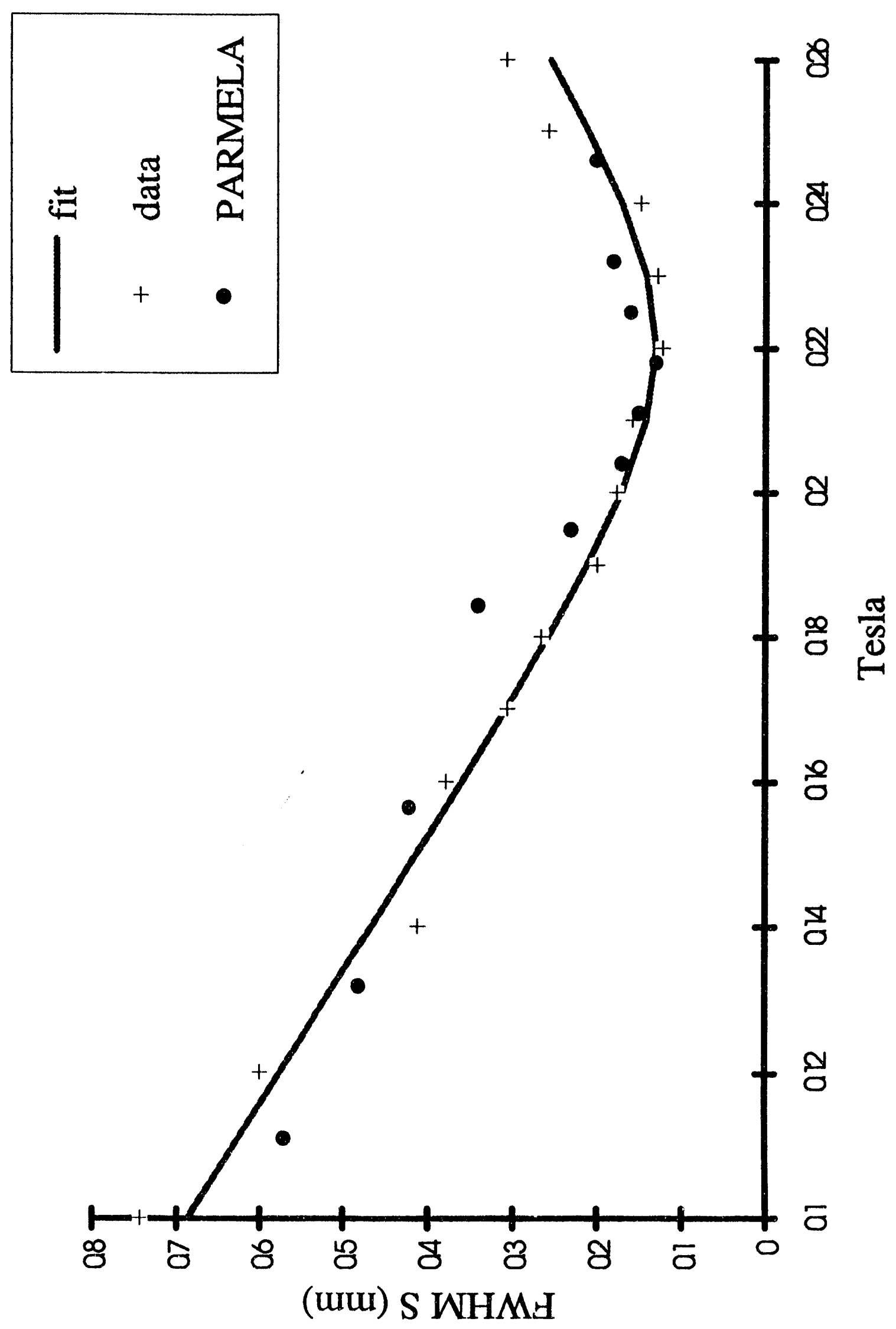




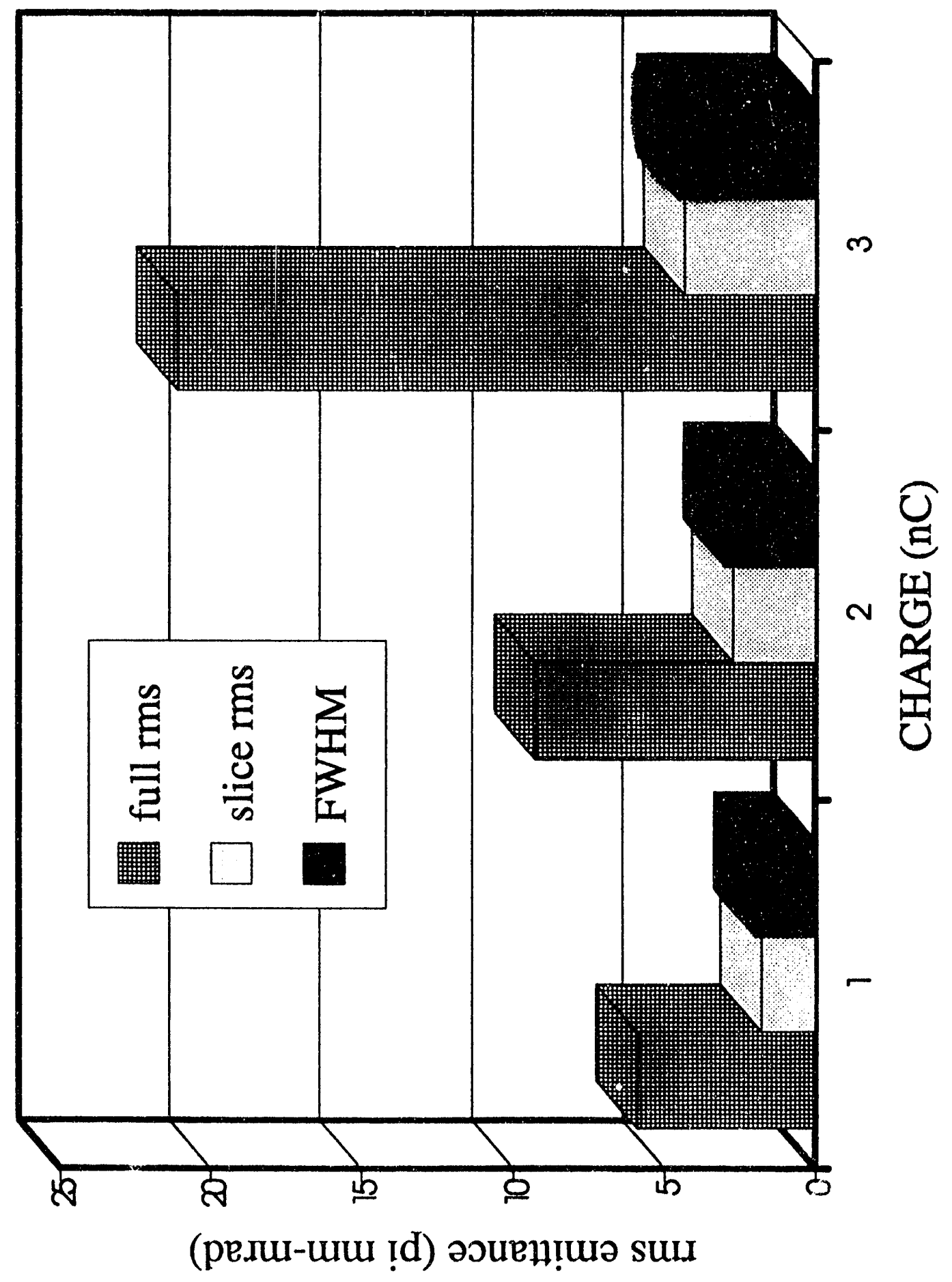

咅 


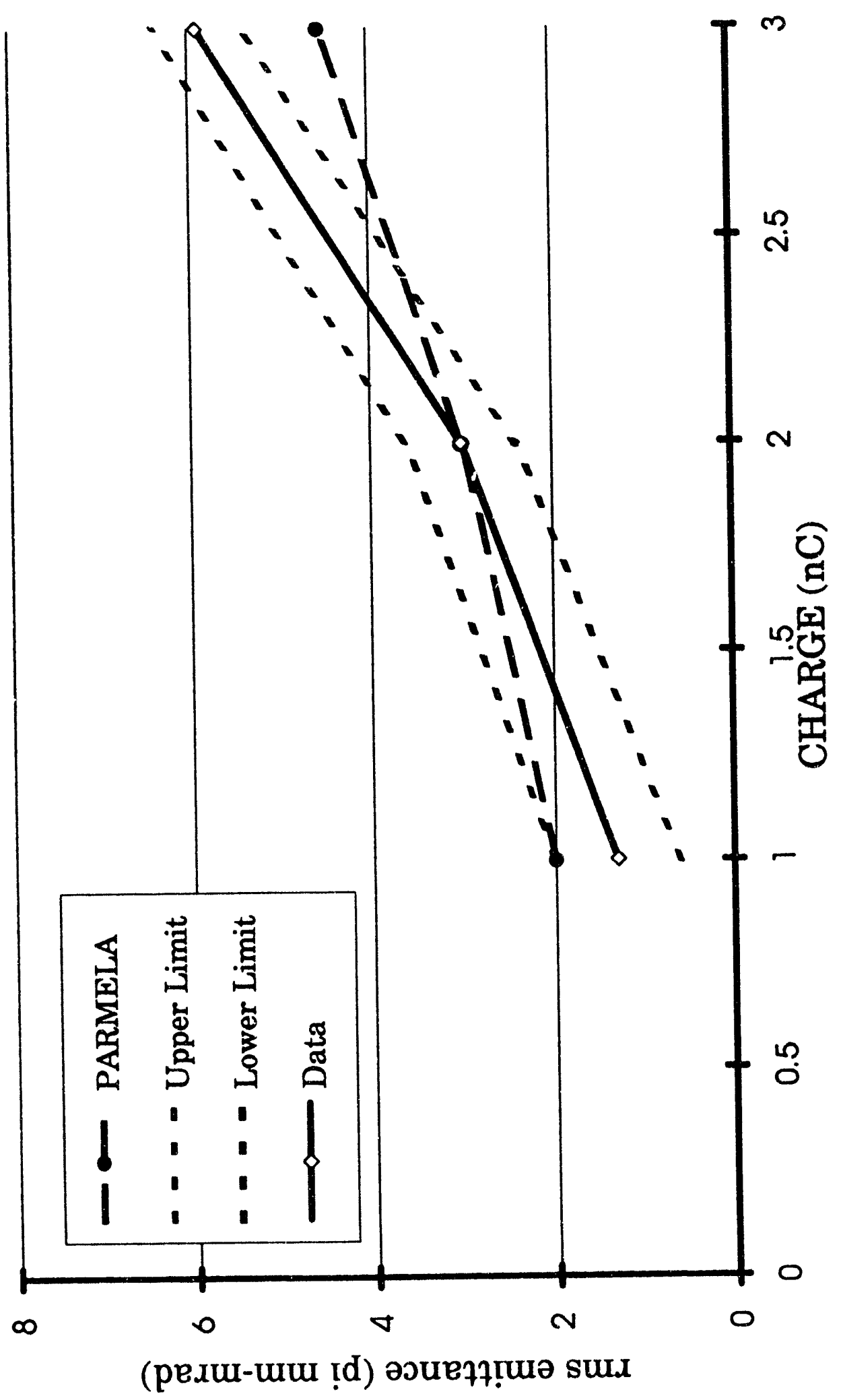

品 
Figure 1. AFEL linac schematic. The focusing solenoid provides compensation of space-charged induced emittance growth. The Bucking Solenoid is adjusted to give zero field at the surface of the cathode. The LINAC is an eleven-cell pi/2-mode structure. The LINAC can be operated from 77K to greater than $400 \mathrm{~K}$. The support structures allow only rotation of the accelerator on cool-down, not translation.

Figure 2. Placing a solenoid near the cathode can compensate for space-charge emittance growth. Position 1 is at the cathode. Positions' 2 and 3 are before and after the solenoid center (with the center between 5 and $15 \mathrm{~cm}$ from the cathode). Position 4 is downstream of the accelerator. The figure also shows the growth in emittance ( $1.5 \pi \mathrm{mm}$-mrad) above the thermal emittance $(1.25 \pi \mathrm{mm}$-mrad) present at the cathode.

Figure 3. Experimental and simulation data for the $1 \mathrm{nC}$ emittance measurement. The simulation data has statistical uncertainty due to the finite number of particles and the distribution of simulation points into bins. The solid curve is a fit to a quadrupole scan function that gives the beam emittance and other beam characteristics.

Figure 4. Comparison of PARMELA emittances calculated for the whole beam (full $\mathrm{rms}$ ), for the rms emittance of a slice (slice rms), and for the FWHM measured from the plot of beam particle number versus transverse dimension (FWHM).

Figure 5. Comparison of experimental and simulation results for 1, 2, and $3 \mathrm{nC}$. The upper and lower limit traces are error bars due mainly to shot-to-shot variations and the minimum resolution of the OTR screen. 

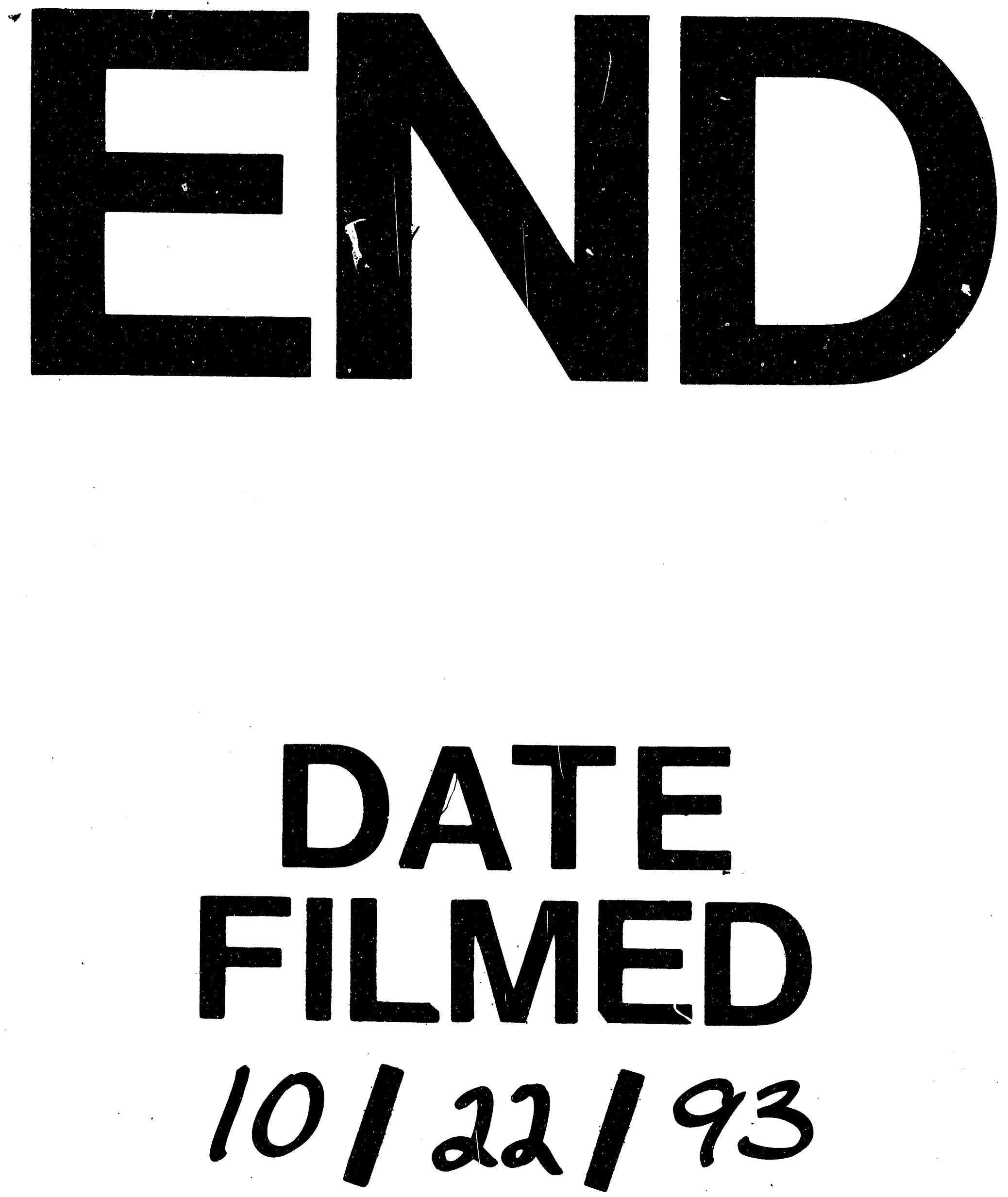

1 
